\title{
Gerenciamento de resíduos de serviços de saúde em aldeias indígenas maranhenses
}

\author{
Evaluation of waste management of health services generated in indigenous health care \\ Evaluación de la gestión de residuos de los servicios de salud generados en la atención sanitaria
}

indígena

Recebido: 31/01/2022 | Revisado: 05/02/2022 | Aceito: 13/02/2022 | Publicado: 18/02/2022

\author{
Leeds Queiroz de Vilar Aleixo \\ ORCID: https://orcid.org/0000-0002-3273-790X \\ Secretaria Municipal de Saúde de São Luís, Brasil \\ E-mail: leedsvilar@gmail.com \\ Risete Maria Queiroz Leão Braga \\ ORCID: https://orcid.org/0000-0003-4267-7426 \\ Universidade Federal do Pará, Brasil \\ E-mail: risetemaria@yahoo.com.br
}

\begin{abstract}
Resumo
O estudo teve como objetivo avaliar o gerenciamento dos Resíduos de Serviço de Saúde (RSS) em Terras Indígenas no Maranhão. A metodologia do estudo consistiu em acompanhar "in loco", no período de janeiro a dezembro de 2018, o atendimento à saúde indígena e os procedimentos do gerenciamento dos RSS gerados nas aldeias maranhenses. Inicialmente, foi realizada uma segregação e classificação desses resíduos de acordo com a RDC $n^{\circ}$ 222/2018 da ANVISA. Em seguida quantificaram-se os RSS, por classe, e identificou-se o tratamento, destinação e disposição final que são dados aos mesmos. Os resultados apontaram que, grande parte dos RSS gerados se se enquadram no Grupo A, com um quantitativo total de $1.314 \mathrm{~kg}$. Quanto ao tratamento os resíduos classificados nos grupos A1, B e E, todos são encaminhados para incineração. Ressalta-se que, a destinação e a disposição final estão corretas quando existe Unidades Básicas de Saúde Indígena (UBSI) implantadas nas aldeias. Entretanto, os RSS gerados nas aldeias que não possuem essas unidades, apresentam a etapa de segregação limitada e problemática, o que implica em um tratamento e disposição ambientalmente comprometida. A elaboração e implantação de Plano de Gerenciamento de Resíduos de Serviços de Saúde (PGRSS) distintos, para as unidades e aldeias, e um programa de educação permanente especializado para as equipes multidisciplinares de saúde indígena (EMSIs) parece ser uma alternativa viável para melhorar o processo de gestão de RSS nessas aldeias que não possuem UBSI, mas que recebem atendimento de saúde em suas ocas.
\end{abstract}

Palavras-chave: Resíduos de serviço de saúde; Gerenciamento; Aldeias indígenas.

\begin{abstract}
The study aimed to evaluate the management of Health Service Waste (RSS) in Indigenous Lands in Maranhão. The study methodology consisted of monitoring, from January to December 2018, the indigenous health care and the procedures for managing the RSS generated in the villages of Maranhão. Initially, a segregation and classification of these wastes was carried out in accordance with ANVISA's RDC $\mathrm{n}^{\circ} .222 / 2018$. Then, the RSS were quantified, by class, and the treatment, destination and final disposal that are given to them were identified. The results showed that most of the generated RSS fall into Group A, with a total amount of 1,314 kg. As for the treatment of waste classified in groups A1, B and E, all are sent for incineration. It is noteworthy that the destination and final disposal are correct when there are Basic Indigenous Health Units (UBSI) implanted in the villages. However, the RSS generated in villages that do not have these units, present the stage of limited and problematic segregation, which implies an environmentally compromised treatment and disposal. The elaboration and implementation of a separate Health Services Waste Management Plan (PGRSS) for the units and villages, and a specialized permanent education program for the multidisciplinary indigenous health teams (EMSIs) seems to be a viable alternative to improve the process of RSS management in these villages that do not have UBSI, but that receive health care in their huts.
\end{abstract}

Keywords: Health service waste; Management; Indigenous villages.

\section{Resumen}

El estudio tuvo como objetivo evaluar la gestión de los Residuos de los Servicios de Salud (RSS) en Tierras Indígenas de Maranhão. La metodología del estudio consistió en monitorear "in loco", de enero a diciembre de 2018, la atención a la salud indígena y los procedimientos de gestión de las RSS generadas en las aldeas de Maranhão. Inicialmente, se realizó una segregación y clasificación de estos residuos de acuerdo con la RDC N ${ }^{\circ}$ 222/2018 de ANVISA. Luego, se cuantificaron las RSS, por clase, y se identificó el tratamiento, destino y disposición final que se les da. Los resultados mostraron que la mayor parte de los RSS generados pertenecen al Grupo A, con una cantidad total de $1.314 \mathrm{~kg}$. En 
cuanto al tratamiento de los residuos clasificados en los grupos A1, B y E, todos se envían a incineración. Se destaca que el destino y disposición final son correctos cuando existen Unidades Básicas Indígenas de Salud (UBSI) implantadas en las veredas. Sin embargo, las RSS generadas en veredas que no cuentan con estas unidades, presentan la etapa de segregación limitada y problemática, lo que implica un tratamiento y disposición ambientalmente comprometida. La elaboración e implementación de un Plan de Manejo de Residuos de los Servicios de Salud (PGRSS) separado para las unidades y veredas, y un programa de educación permanente especializada para los equipos multidisciplinarios de salud indígena (EMSI) parece ser una alternativa viable para mejorar el proceso de gestión de los RSS en estos pueblos que no tienen UBSI, pero que reciben atención médica en sus chozas.

Palabras clave: Residuos de servicios de salud; Gestión; Pueblos indígenas.

\section{Introdução}

A disposição inadequada dos resíduos sólidos no meio ambiente vem sendo apontada como um dos mais graves problemas ambientais da atualidade na maioria dos municípios brasileiros. Geralmente, após o fechamento, essas áreas degradadas necessitam de uma remediação e de recuperação ambiental (Brasil, 2010). No entanto, são as pequenas comunidades que requerem maiores orientações sobre os métodos adequados de destinação final, respeitando suas realidades, no que diz respeito aos aspectos de projeto, execução, operação, monitoramento ambiental e inter-relação cultural.

A Associação Brasileira de Empresas de Limpeza Pública e Resíduos Especiais (ABRELPE, 2019) revela que, em 2018, foram geradas no Brasil 79 milhões de toneladas de resíduos sólidos, um aumento de pouco menos de $1 \%$ em relação ao ano anterior. Deste montante, 92\% (72,7 milhões de toneladas) foram coletados, evidenciando que 6,3 milhões de toneladas de resíduos não foram recolhidas junto aos locais de geração. A ABRELPE (2019) cita que a destinação adequada em aterros sanitários recebeu 59,5\% dos resíduos sólidos urbanos (RSU) coletados, ou seja, 43,3 milhões de toneladas, que foi considerado um pequeno avanço em relação ao cenário do ano anterior. O restante (40,5\%) foi despejado em locais inadequados por 3.001 municípios, o que representa 29,5 milhões de toneladas de RSU destinados aos lixões ou aterros controlados, que não contam com um conjunto de sistemas e medidas necessários para proteger a saúde das pessoas e o meio ambiente contra danos e degradações.

Gomes (2012) relata que uma categoria de resíduos que merece atenção, devido ao seu potencial de risco tanto a saúde humana quanto ao meio ambiente, são os Resíduos de Serviços de Saúde (RSS), em razão de suas características tóxicas e/ou patogênicas. Quando gerenciados de forma inadequada, impactam negativamente o ambiente, devido suas características químicas, biológicas e físicas (Seraphim, 2016).

Estes impactos são ainda maiores nas populações indígenas, por serem consideradas mais vulneráveis ao acometimento de doenças, além de não possuir, na prática, acesso ao saneamento quanto a responsabilidades de âmbito municipal e estadual, nas quais estão inseridas (Athias \& Machado, 2001).

Assim, esses resíduos, demandam um gerenciamento específico, necessitando de cuidados especiais desde o momento da geração até a disposição final, o que requer legislações cada vez mais restritivas, para garantir essa disposição segura e adequada (Seraphim, 2016).

Nas aldeias, esses resíduos são gerados através do atendimento a saúde, baseado em um modelo assistencial aos povos indígenas que está configurado através de uma rede de serviços implantado nas terras indígenas, a partir de critérios geográficos, demográficos e culturais (Brasil, 2002). No entanto, os RSS gerados através desta assistência, constituem um problema sob dois aspectos: o intra estabelecimento, controle da gestão de resíduos gerados dentro de um estabelecimento de saúde (ABNT, 1993a); e, o extra estabelecimento, que se refere ao gerado durante assistência à saúde aldeia a aldeia, isto é, estratégia de atendimento realizada de acordo com os preceitos do Sistema Único de Saúde brasileiro (SUS). Nesse último, a equipe de saúde da família compõe um processo de trabalho com maior potencial de aprofundar os princípios, diretrizes e fundamentos da atenção básica, através de uma visita domiciliar (Brasil, 2007).

Tanto os resíduos gerados intra quanto o extra estabelecimento possuem composição e características propícias à 
sobrevivência e proliferação de organismos patogênicos (ABNT, 1993), bem como para a constituição de ambiente ecológico favorável à proliferação de certos animais, que podem se tornar fontes ou reservatórios de agentes causadores de enfermidades à coletividade (Lippel, 2003).

Observando tal perspectiva, apesar de não serem "naturalmente ecologistas", os índios têm consciência da sua dependência - não apenas física, mas, sobretudo cosmológica - em relação ao meio ambiente (Silva, 2017). Assim, diante desta realidade, realizou-se um estudo, com base na legislação vigente, para avaliar as etapas de descarte, tratamento e destinação dos RSS, gerados nos atendimentos de saúde nas aldeias indígenas do Maranhão. O trabalho visou fornecer ferramentas técnicas para a proposição de um Plano de Gerenciamento de Resíduos de Serviços de Saúde (PGRSS) futuramente, vinculado a uma rotina de ação em saúde nestas aldeias, buscando responder à questão-problema: Como é realizado o manejo dos resíduos sólidos dos serviços de saúde nas aldeias haja vista o seu difícil acesso?

\section{Metodologia}

\section{Área de estudo}

O trabalho foi desenvolvido nas terras indígenas na abrangência do Estado do Maranhão, em uma área territorial de 19.083,83 km² a qual concentra a $7^{\text {a }}$ maior população indígena do Brasil (Freitas, 2013).

As ações do SasiSUS no Maranhão estão organizadas na forma de seis Polos Base, tendo suas sedes localizadas nos municípios de Amarante, Arame, Barra do Corda, Grajaú, Santa Inês e Zé Doca (Cantuária, 2020). Estas ações possuem o objetivo de atender, conforme o DSEI-MA (2018), a 33.878 índios das etnias Guajajara, Kaapor, Gavião, Awá Guaja, Kreniê, Krikati e Kanela, localizados nas regiões dos polos de Grajaú, Barra do Corda, Arame, Santa Inês, Zé Doca e Amarante, distribuídos em 626 aldeias, que se encontram localizadas em 23 municípios maranhenses (Figura 1). 
Figura 1. Abrangência do Distrito Sanitário Especial Indígena do Maranhão.

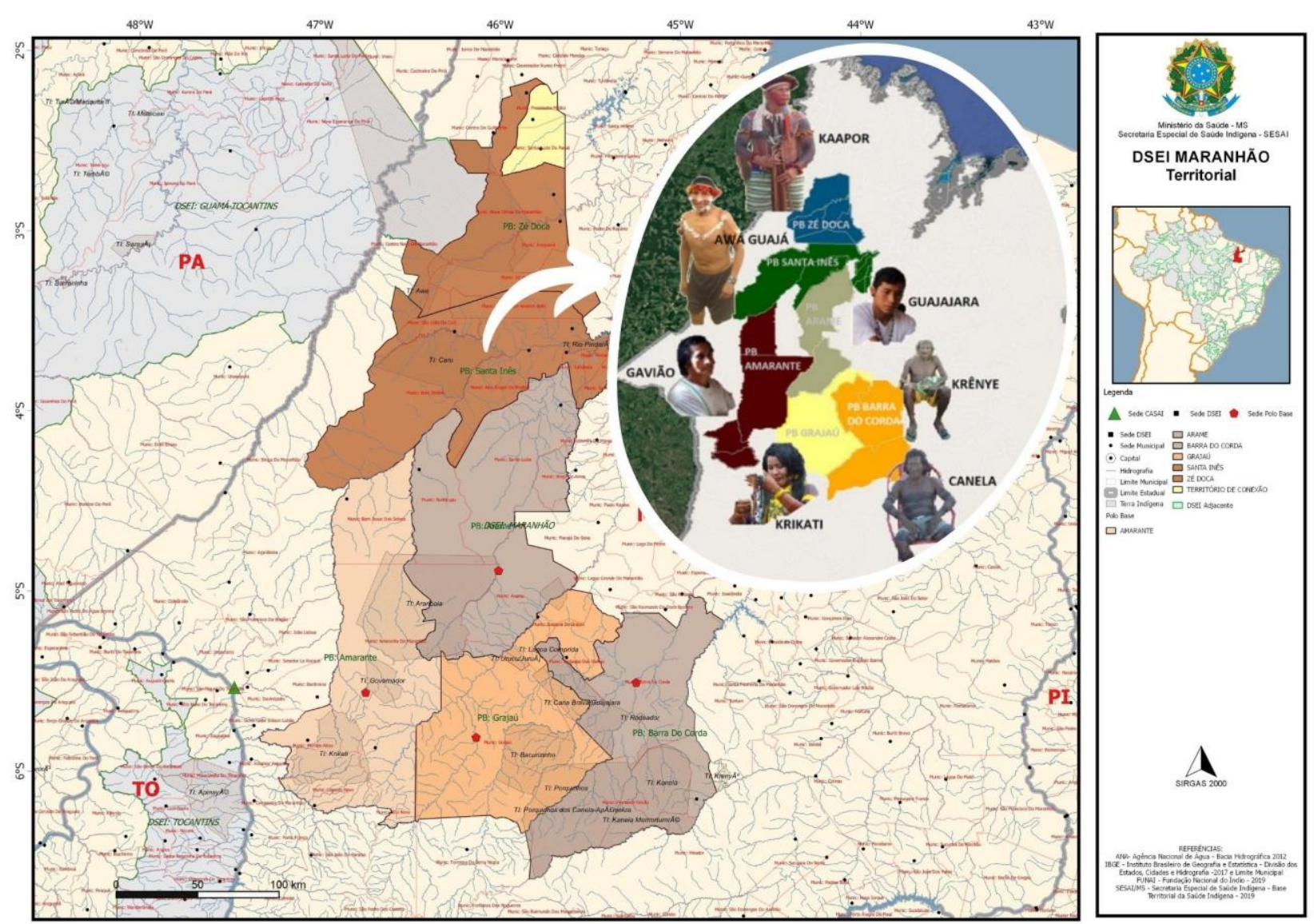

Fonte: Autores (2020).

Na Figura 1 observa-se a disposição dos polos bases no estado do Maranhão, considerando as etnias existentes de referencia em cada polo.

Nas terras indígenas foram identificadas 49 (quarenta e nove) Unidades Básicas de Saúde Indígena (UBSI), pertencentes à estrutura de atendimento ao indígena, que se caracterizam como a atenção básica ou atenção primária em saúde, também conhecida como a "porta de entrada" dos usuários indígenas nos sistemas de saúde.

\section{Etapas metodológicas}

O trabalho foi dividido em 3 (três) etapas tendo como abordagem uma pesquisa quali-quantitativa descritiva, ou seja, trata-se de uma pesquisa que se preocupa com a análise do mundo empírico em seu ambiente natural, valorizando o contato direto e prolongado do pesquisador com o ambiente e a situação diretamente estudada (Godoy, 1995).

A $1^{\text {a }}$ etapa consistiu na caracterização das áreas de atendimento nas aldeias indígenas maranhenses com relação à descrição do modelo de atendimento à saúde indígena, a fim de identificar os tipos de resíduos gerados e a sua correta gestão nas terras indígenas do Estado do Maranhão. $\mathrm{Na} 2^{\mathrm{a}}$ etapa foram caracterizados em todos os postos de saúde identificados, através de um acompanhamento diário em campo, no período de janeiro a dezembro de 2018, os procedimentos do gerenciamento dos RSS adotados nas aldeias, além da quantificação de profissionais disponíveis e a qualificação dos serviços prestados. Por tratar-se de uma pesquisa qualitativa, com enfoque em um estudo de caso, foi empregada como instrumento de coleta de dados a observação participante no atendimento das equipes de saúdes nas aldeias indígenas maranhenses, buscando mostrar a realidade percebida, explanando principalmente as não conformidades encontradas durante as etapas funcionais do gerenciamento dos RSS (segregação, quantificação, acondicionamento, coleta interna e transporte, armazenamento e coleta 
externa). Nesta etapa foi realizada a caracterização quali-quantitativa dos RSS gerados (geração, segregação e classificação por tipologia de RSS). A caracterização qualitativa foi obtida por meio de amostragens representativas baseadas na classificação dos RSS, descritos pela ANVISA (2018), através da Resolução de Diretoria Colegiada no 222.

$\mathrm{Na} 3^{\text {a }}$ etapa foi avaliado o aspecto do gerenciamento dos RSS gerados quanto a segregação por classes, coleta, tratamento, destinação e disposição final, e comparado ao previsto no Plano de Gerenciamento de Resíduos de Serviço de Saúde (PGRSS) nos PBs e UBSIs (Almeida, 2014).

\section{Resultados e Discussão}

\section{Modelo de atendimento de saúde dos indígenas}

Para identificar o modelo de atendimento de saúde dos indígenas foi realizada uma avaliação de dados entre os serviços de saúde realizados nas aldeias indígenas e os tipos de resíduos gerados em cada atendimento. O modelo de atendimento é operacionalizado, em um primeiro nível, pela atuação das equipes multidisciplinares de saúde indígena (EMSIs) nas aldeias, sendo formadas por enfermeiros, técnicos de enfermagem, odontólogos, médicos, agentes de saúde bucal, agentes indígenas de saúde e de saneamento (Brasil, 2017).

As atividades são desenvolvidas em todo o território indígena maranhense tanto para as aldeias que possuem postos de saúde como também as que não possuem. No entanto, todos os dados geridos de atendimento e geração de RSS referem-se apenas aos atendimentos ocorridos nas UBSIS, não sendo contabilizados os atendimentos realizados diretamente nas ocas, com geração de resíduos equivalentes. Na Tabela 1 está apresentada a relação de polos base existentes, a quantidade de indígenas e equipes de saúde e as USBI, bem como a quantidade de aldeias por bolo base. Destaca-se também a quantidade per capita de atendimento indígena por Polo Base (Tabela 1).

Tabela 1. Distribuição das EMSIs do DSEI-MA em 2018.

\begin{tabular}{lccccc}
\hline \multicolumn{1}{c}{ POLO BASE } & $\begin{array}{c}\text { Quantidade de } \\
\text { Indígenas }\end{array}$ & $\mathrm{N}^{\circ}$ EMSI & Quantidade de UBSI & $\begin{array}{c}\text { Quantidade de } \\
\text { aldeias }\end{array}$ & $\begin{array}{c}\text { Per capita de indígenas } \\
\text { atendidos/EMSI }\end{array}$ \\
\hline AMARANTE & 6.458 & 7 & 7 & 95 & 922,57 \\
ARAME & 4.926 & 3 & 7 & 89 & 1.642 \\
BARRA DO CORDA & 11.339 & 11 & 17 & 243 & $1.030,82$ \\
GRAJAÚ & 7.268 & 6 & 7 & 168 & $1.211,33$ \\
SANTA INÊS & 1.842 & 3 & 6 & 18 & 614 \\
ZÉ DOCA & 1.691 & 2 & 49 & 626 & 845,5 \\
TOTAL & 33.524 & 32 & 6 & \\
\hline
\end{tabular}

Fonte: Autores (2019).

Trabalham nas áreas indígenas 32 EMSIs, distribuídas nos seis Polos Base, que desenvolvem as ações da atenção básica à saúde indígena. A maior quantidade de equipes se concentra no Polo Barra do Corda com uma per capita aproximada de 1.000 indígenas atendidos. Portanto, considerando o preconizado pela Portaria Federal n ${ }^{\circ}$ 1.088/2005 (BRASIL, 2005), que orienta a média per capta de 500 indígenas atendidos por equipe, verifica-se um déficit de numero de EMSI para todos os Polos Base.

Os atendimentos efetivados a saúde dos indígenas foram considerados somente nas 49 UBSIS existentes, sendo verificadas as etapas de segregação, acondicionamento, destinação (tratamento) e disposição final dos RSS, de acordo com a RDC n 222, (ANVISA, 2018). 


\section{Geração, segregação e classificação por tipologia de RSS}

Os RSS gerados, no período de janeiro a dezembro de 2018, em atendimento aos indígenas pelas EMSIs foram coletados, segregados e encaminhados aos 6 (seis) Polos Base maranhenses. A segregação ocorreu por grupos e subgrupos de RSS (ANVISA, 2018):

- Grupo A - Subgrupo A1, resultantes de atividades de vacinação com microrganismos vivos atenuados e/ou inativados (frascos de vacinas com expiração do prazo de validade, com conteúdo inutilizado ou com restos do produto e seringas, quando desconectadas);

- Grupo A - Subgrupo A4, recipientes e materiais resultantes do processo de assistência à saúde (sondas, curativos, algodão usado, luvas de procedimento e mascaras);

- Grupo B - insumos farmacêuticos, oriundos dos restos de medicamentos utilizados no atendimento;

- Grupo E - materiais perfurocortantes, gerados através da assistência medica e odontológica.

Os resíduos do Grupo A foram acondicionados em sacos brancos leitosos, e posteriormente em bombonas identificadas. Os resíduos do Grupo B, medicamentos vencidos, foram colocados em caixas de papelão. E os resíduos do Grupo E foram imediatamente acondicionados em caixas tipo descartex. A quantidade gerada de RSS por tipologia, número de atendimentos pela equipe, e a per capita por atendimento dos indígenas estão apresentadas na Tabela 2.

Tabela 2. Quantidades e per capita de RSS dos Grupos A, B e E em atendimento aos indígenas em 2018.

\begin{tabular}{l|c|c|c|c|c|c|c}
\hline \multirow{2}{*}{ PB } & Atendimentos & \multicolumn{2}{c}{ RSS - Grupo A } & \multicolumn{2}{c}{ RSS - Grupo B } & \multicolumn{2}{c}{ RSS - Grupo E } \\
\cline { 3 - 8 } & & $(\mathrm{kg})$ & $\mathrm{q}$ & $(\mathrm{kg})$ & $\mathrm{q}$ & $(\mathrm{kg})$ & $\mathrm{q}$ \\
\hline Amarante & 923 & 360 & 0,39 & 15 & 0,02 & 42 & 0,05 \\
\hline Arame & 704 & 144 & 0,20 & 33 & 0,05 & 48 & 0,07 \\
\hline Barra do Corda & 1.620 & 279 & 0,17 & 222 & 0,14 & 154 & 0,10 \\
\hline Grajau & 1.038 & 261 & 0,25 & 0 & 0,00 & 38 & 0,04 \\
\hline Santa Inês & 263 & 117 & 0,44 & 6 & 0,02 & 25 & 0,10 \\
\hline Zé Doca & 242 & 153 & 0,63 & 36 & 0,15 & 3 & 0,01 \\
\hline TOTAL DE RSS RECEBIDOS DE TODOS OS PB (kg) & 1.314 & & 312 & & 310 & \\
\hline
\end{tabular}

Fonte: Autores (2019).

Foram identificados e classificados no Grupo A um total de $1.314 \mathrm{~kg}$ de RSS, sendo o Polo Base (PB) de Amarante o que mais recebeu esse tipo de resíduo das aldeias, $360 \mathrm{~kg}$. O PB Barra do Corda e PB Grajau receberam $279 \mathrm{~kg}$ e $261 \mathrm{~kg}$, respectivamente.

Nota-se que os Polos Base de Barra do Corda, Amarante, e Grajaú apresentaram uma quantidade maior de indígenas e um menor número de EMSIs por atendimento quando comparado aos Polos Base de Santa Inês e Zé Doca, ou seja, existe uma defasagem de EMSI de um polo para outro (Tabela 1). Portanto, a grande variação da quantidade de resíduos em cada mês por PB pode estar relacionada à falta de atendimento em uma determinada região.

É importante ressaltar que o adensamento populacional, as características de acesso às aldeias da região, acabam por definir um maior número de procedimentos em cada atendimento ao indígena. Existem acessos, que a distância em relação ao DSEI-MA as aldeias são quase $700 \mathrm{~km}$, os quais são realizados por transporte fluvial e terrestre. Logo, as EMSIs que trabalham em aldeias onde o acesso é mais difícil acabam realizando o maior número possível de atendimentos e serviços.

Também foram identificados restos de plantas, como resíduos do grupo A, que são utilizados como curativos em feridas dos indígenas e que não são encaminhados aos PB, sendo simplesmente descartados diretamente na superfície nas 
aldeias indígenas.

Com relação aos RSS dos grupos B os dados coletados revelaram que, o PB de Barra do Corda recebeu uma maior quantidade desses resíduos em relação aos demais polos. Para os resíduos caracterizados como restos de produtos químicos, medicamentos vencidos e parcialmente utilizados, tornando-os sem uso, foi identificado um possível modelo de dispensação de medicamentos em função da organização de cada EMSI.

Para os RSS identificados como Grupo E verifica-se também que o PB Barra do Corda foi o que mais recebeu resíduos perfurocortantes em relação aos demais polos. Isso ocorreu em função da maior quantidade de equipes e o maior número de indígenas atendidos. Os PBs de Santa Inês e Zé Doca receberam $263 \mathrm{~kg}$ e $242 \mathrm{~kg}$ de resíduos, respectivamente o que pode caracterizar poucos atendimentos realizados.

Em resumo, a maior quantidade de RSS gerados nas aldeias foi classificada como sendo grupo A. É possível constatar que a maior parte foi proveniente de atendimentos em aldeias situadas no PB Amarante. Entretanto, o maior número de atendimentos foi nas aldeias do PB Barra do Corda, com menor geração de RSS, como pode ser verificado a partir da per capita de atendimento ao ano (q).

Este resultado pode estar relacionado ao tipo de atendimento recebido em cada polo. Considerando que os resíduos do Grupo A correspondem a ampolas e seringas (restos de vacinas), algodão usado, luvas de procedimento e mascaras, pode-se afirmar que indígenas do PBs de Santa Inês e Zé Doca, de acordo com o valor q, receberam mais vacinas do que os indígenas dos outros polos, e que podem estar recebendo também mais atendimentos eletivos, como consultas médicas assistenciais, nutricionais e psicológicas. Isto pode ocorrer, devido às etnias existentes em cada terra indígena.

Em Santa Inês estão localizados os indígenas de recente contato, como por exemplo, os Awá guajás, que demandam maior atenção da equipe de saúde, devido à baixa imunidade.

Para os resíduos do Grupo B a maior quantidade de RSS foi recebida no PB Barra do Corda, devido a grande quantidade de atendimentos, refletida pelo maior adensamento populacional indígena. No entanto, considerando o valor q, o PB Zé Doca recebeu das EMSIs mais medicamentos vencidos ou avariados do que os demais polos, o que pode ser caracterizado pela forma de atendimento realizada. Neste polo, as equipes permanecem mais tempo nas aldeias do que em outros polos em função do acesso. Considerando que os tipos de resíduos do Grupo E, concentram-se em materiais perfurocortantes, provenientes da assistência médica e odontológica, pode-se afirmar que os indígenas do PB de Santa Inês, de acordo com o valor q, recebem mais atendimento odontológico, do que os indígenas dos outros polos.

A Figura 2 resume a quantidade total de resíduos gerados, por grupo, em assistência a saúde indígena pelas EMSI nos polos base no ano de 2018. 
Figura 2. Quantidade de RSS gerados em 2018, por Grupo, em atendimento aos indígenas pelas EMSIs nas UBSIS.

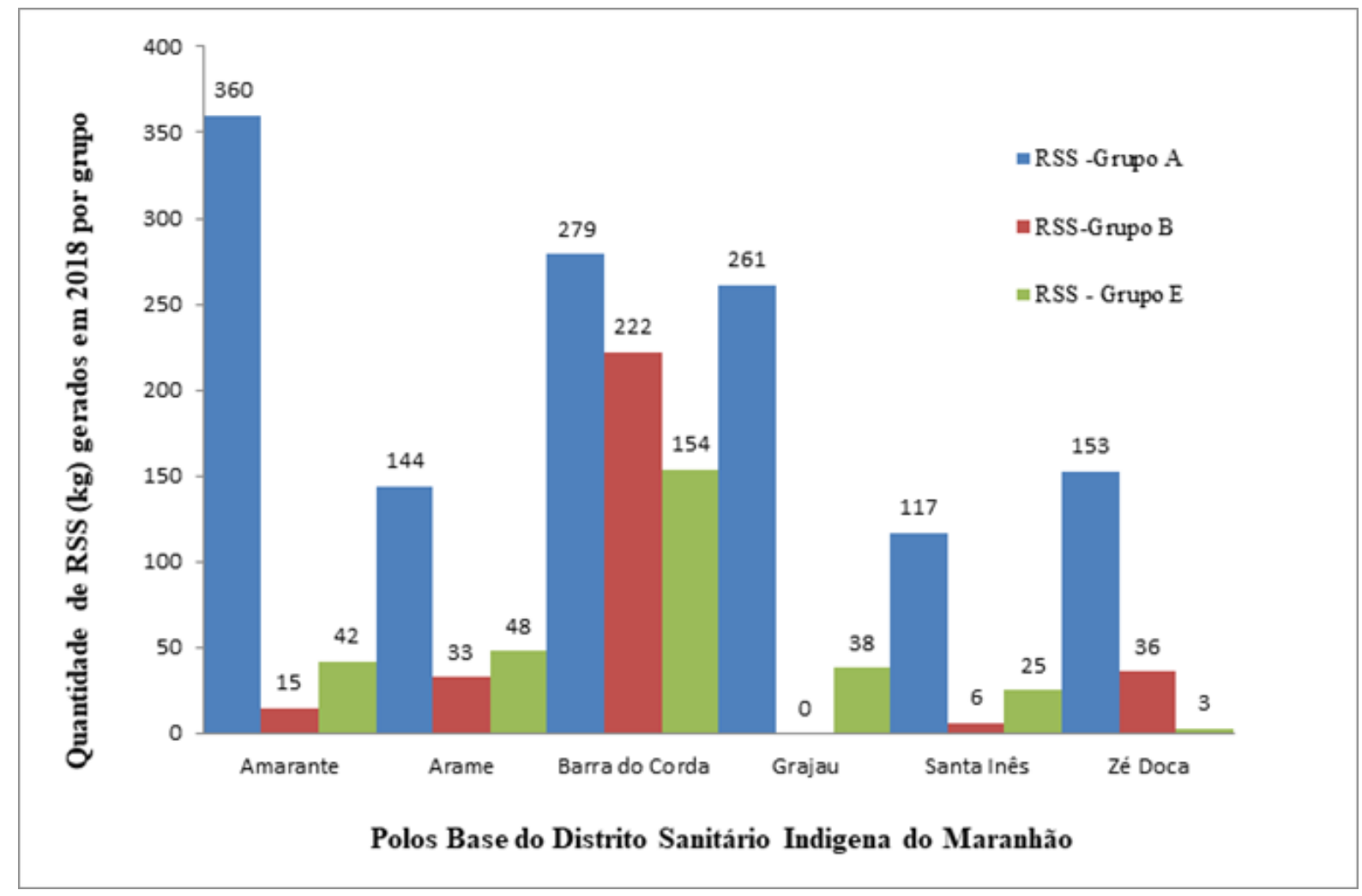

Fonte: Autores (2019).

\section{Tratamento, destinação e disposição final.}

Quanto ao tratamento e à disposição final, a Resolução no 358, CONAMA (2005), e a Resolução n 283 , CONAMA (2001), estabelece que, alguns tipos de resíduos devem ser tratados ou destinados de formas diferenciadas de acordo com a sua classificação. Conforme observado, a empresa contratada pelo DSEI-MA realiza o encaminhamento dos RSS com risco biológico - Grupo A4 para unidades de incineração. Os resíduos do grupo A1 e B são encaminhados para autoclavagem e em seguida são incinerados. Os resíduos do Grupo E, acondicionados em caixas descartex, são encaminhados para incineração. Após tratamento, todo o rejeito dos tratamentos é encaminhado para a disposição final na central de gerenciamento ambiental Titara, aterro sanitário Classe I, localizado na Fazenda Arapixi, s/n, Zona Industrial de Rosário - MA.

Ao realizar um cruzamento de dados com o previsto no Plano de Gerenciamento de Resíduos de Serviço de Saúde (PGRSS) existente nas sedes dos PBs, o qual abrange os postos de saúde, e a informação de acondicionamento diferenciado nas aldeias, por meio dos atendimentos realizados pela equipe de saúde, nota-se que:

- os PGRSS dos PBs e UBSIs, considerando a RDC 222, (ANVISA, 2018), a estimativa diária da quantidade dos RSS gerados por grupos A, B, e E - logo se verifica que esse procedimento é realizado nos PBs, bem como os relacionados à geração, segregação por identificação de classes, acondicionamento, coleta, armazenamento, transporte, tratamento e à disposição final;

- para as aldeias que não possuem UBSIs implantadas e, portanto, sem PGRSS, mas que recebem ações de saúde pelas EMSI em atendimento volante (casa a casa), nota-se a ausência de um gerenciamento adequado dos RSS gerados durante o atendimento. Constatou-se que os resíduos são descartados diretamente ao solo, revelando assim a necessidade de implantação de um PGRSS por área de atuação das EMSIs. 


\section{Conclusão}

O modelo assistencial à saúde aos povos indígenas está configurado através de uma estratégia de atendimento inicial, realizada de acordo com os preceitos do SUS brasileiro, no qual a equipe de saúde da família compõe um processo de trabalho com maior potencial de aprofundar os princípios, diretrizes e fundamentos da atenção básica, através da visita domiciliar. Estas redes de serviços funcionam nas terras indígenas, a partir de critérios geográficos, demográficos e culturais apresentados.

Considerando a RDC 222 da ANVISA (2018) e as etapas do gerenciamento de RSS, nas aldeias indígenas maranhenses, foi estimada uma quantidade de quase duas toneladas de RSS gerados, e que foram classificados nos grupos A, B, e E, no atendimento a saúde indígena no ano de 2018, nas 49 unidades básicas de saúde indígena.

A quantidade de RSS segregados e enviados para a coleta especializada ocorre nas aldeias que possuem UBSI implantadas, nas demais aldeias que não possuem unidades de saúde, o atendimento de saúde pelas EMSI e a geração de resíduos não segue um gerenciamento adequado, o que pode comprometer a qualidade de vida dos indígenas e promover um forte impacto ambiental, decorrente da poluição do meio ambiente, visto o descarte desses resíduos em terrenos próximos as aldeias.

Logo, faz-se necessário um programa de educação permanente, especializado para as EMSIs, capacitando-as para realizar cada etapa do gerenciamento dos RSS, de acordo com a Resolução 222/2018 da ANVISA nas aldeias que não possuem UBSI. A reflexão sobre modelos de gestão de RSS para atendimento volante deve ser implantada.

Como sugestão, para trabalhos futuros, recomenda-se o desenvolvimento de pesquisas correlacionadas à geração de novos resíduos, como os provenientes do uso de testes rápidos para detecção do COVID-19 nas aldeias indígenas, tendo como embasamento os protocolos de segurança utilizados, as características dos resíduos, suas dificuldades e o contexto em que a prática assistencial se desenvolve, o que pode apontar para a necessidade de uma destinação adequada.

\section{Referências}

ABNT. Associação Brasileira de Normas Técnicas. (1993a). NBR 12808 - Resíduos de Serviços de Saúde: Classificação. https://wp.ufpel.edu.br/residuos/files/2014/04/NBR-12808-1993-Res\%C3\%ADduos-de-servi\%C3\%A7os-de-sa\%C3\%BAde.pdf.

ABNT. Associação Brasileira de Normas Técnicas. (1993). NBR 12809 - Resíduos de Serviços de Saúde: Procedimentos necessários ao gerenciamento intraestabelecimento. https://pt.scribd.com/document/396343483/ABNT-NBR-12809-pdf

ABRELPE. Associação Brasileira de Empresas de Limpeza Pública e Resíduos Especiais (2019). Os descaminhos do lixo. [blog] https://opiniao.estadao.com.br/noticias/notas-e-informacoes,os-descaminhos-do-lixo,70003103935

Almeida, M. (2011). Elaboração de projeto, TCC, dissertação e tese: uma abordagem simples, prática e objetiva. Editora Atlas.

ANVISA. Agência de Vigilância Sanitária. (2018). Resolução de Diretoria Colegiada nº 222, de 28 de março de 2018 que dispõe sobre o Regulamento Técnico para o gerenciamento de resíduos de serviços de saúde. https://bvsms.saude.gov.br/bvs/saudelegis/anvisa/2018/rdc0222_28_03_2018.pdf

Athias, R. \& Machado, M. (2001). A saúde indígena no processo de implantação dos Distritos Sanitários Especiais Indígenas: temas críticos e propostas para um diálogo interdisciplinar. Cadernos de Saúde Pública. 17(2), 425-31. ISSN 0102-311X

Brasil. Fundação Nacional da Saúde. (2002). Política Nacional de Atenção à Saúde dos Povos Indígenas. https://bvsms.saude.gov.br/bvs/publicacoes/politica_saude_indigena.pdf

Brasil. Ministério da Saúde. (2005). Portaria $n^{\circ}$ 1.088, de 04 de julho de 2005 que dispõe sobre a definição dos valores do incentivo financeiro de atenção básica de saúde aos povos indígenas e sobre a composição e organização das equipes multidisciplinares de atenção à saúde indígena. https://bvsms.saude.gov.br/bvs/saudelegis/gm/2005/prt1088_04_07_2005.html

Brasil. Ministério da Saúde. (2007). Portaria nº. 2656, de 17 de outubro de 2007 que dispõe sobre as responsabilidades na prestação da atenção à saúde dos povos indígenas, do Ministério da Saúde e regulamentação dos Incentivos de Atenção Básica e especializada aos Povos Indígenas. https://bvsms.saude.gov.br/bvs/saudelegis/gm/2007/prt2656_17_10_2007_comp.html

Brasil. Ministério da Saúde. (2017). Portaria no 1.317, de 3 de agosto de 2017 que adequa o registro das informações relativas a estabelecimentos que realizam ações de Atenção à Saúde para populações Indígenas no CNES. https://bvsms.saude.gov.br/bvs/saudelegis/sas/2017/prt1317_08_08_2017.html

Brasil. Ministério do Meio Ambiente. (2010). Lei n ${ }^{\circ}$ 12.305, de 02 de agosto de 2010 que institui a Política Nacional de Resíduos Sólidos e dá outras providências. http://www.planalto.gov.br/ccivil_03/_ato2007-2010/2010/lei/112305.htm 
Research, Society and Development, v. 11, n. 3, e21211326352, 2022

(CC BY 4.0) | ISSN 2525-3409 | DOI: http://dx.doi.org/10.33448/rsd-v11i3.26352

Cantuária, A. (2020). Educação permanente e a SESAI: uma análise da política nacional de educação permanente no âmbito da secretaria especial de saúde indígena. 2020. 73 f. Tese (Mestrado em Ciências da Educação) - Universidade Católica Portuguesa, Lisboa.

CONAMA. Conselho Nacional do Meio Ambiente. (2001). Resolução ${ }^{\circ}$ 283, de 12 de julho de 2001 que dispõe sobre o tratamento e a destinação final dos resíduos dos serviços de saúde. https://www.legisweb.com.br/legislacao/?id=97496

CONAMA. Conselho Nacional do Meio Ambiente. (2005). Resolução no 358, de 29 de abril de 2005 que dispõe sobre o tratamento e a disposição final dos resíduos dos serviços de saúde e dá outras providências. https://www.saude.mg.gov.br/images/documentos/res_358.pdf

DSEI-MA. Distrito Sanitário Especial Indígena do Maranhão. (2018). Censo vacinal. https://bvsms.saude.gov.br/bvs/publicacoes/saude_indigena_analise_situacao_sasisus.pdf

Freitas, W. (2013). Cartografia dos Fatores Intervenientes na Mortalidade Materna, Fetal e Infantil nos Distritos Sanitários Especiais Indígenas e dos Itinerários de Produção de Saúde nas Áreas Indígenas. https://www.redehumanizasus.net/sites/default/files/cartografia_dsei_maranhao.pdf

Godoy, A. (1995). Pesquisa qualitativa: tipos fundamentais. Revista de Administração de Empresas, 35(3), 1-10. https://doi.org/10.1590/S003475901995000300004

Gomes, S. (2013). Resíduos sólidos dos serviços de saúde em terras indígenas: o caso do Distrito Sanitário Especial Indígena Médio Rio Purus, Amazonas. 2013. 153 f. Tese (Mestrado em Desenvolvimento Sustentável) - Universidade de Brasília, Brasília.

Lippel, M. (2003). Modelo de Gerenciamento de Resíduos Sólidos de Saúde para Pequenos Geradores - o caso de Blumenau/SC. 2003. 123 f. Tese (Mestrado em Engenharia de Produção) - Universidade Federal de Santa Catarina, Blumenau.

Seraphim, C. R. U. M., Ribeiro, M. L., Sossae, F. C., \& Araújo, D. dos S. (2016). Abordagem dos resíduos de serviços de saúde (RSS) na formação profissional dos auxiliares e técnicos em enfermagem de Araraquara - SP. Revista Brasileira Multidisciplinar, 19(2), 33-48. https://doi.org/10.25061/25272675/ReBraM/2016.v19i2.411

Silva, S. (2017). A ação do serviço à atenção da população indígena nas Casais e Dseis: um olhar da psicologia da saúde. 2017.90 f. Tese (Mestrado em Psicologia) - Universidade Católica Dom Bosco, Campo Grande. 